\title{
Erratum to: Quantitative physiology and elemental composition of Kluyveromyces lactis CBS 2359 during growth on glucose at different specific growth rates
}

\author{
Oscar Dias - Thiago O. Basso (1) Isabel Rocha - Eugénio C. Ferreira • \\ Andreas K. Gombert
}

Published online: 12 October 2017

(C) Springer International Publishing AG 2017

\section{Erratum to: Antonie van Leeuwenhoek DOI 10.1007/s10482-017-0940-5}

In the original publication of the article, the below mentioned errors have appeared. The correct text is provided in this erratum.

In the abstract section, the sentence "This dataset serve" should be replaced as "This dataset serves".

Also, the reference "Basso TO, Gomes FS, Lopes ML, et al (2014) Homo- and heterofermentative lactobacilli differently affect sugarcane-based fuel ethanol fermentation. Antonie Van Leeuwenhoek 105:169-177. doi:10.1007/s10482-013-0063-6" should be replaced as "Basso TO, Dario MG, Tonso A, Stambuk BU, Gombert AK (2010) Insufficient uracil supply in fully aerobic chemostat cultures of Saccharomyces cerevisiae leads to respiro-fermentative metabolism and double nutrientlimitation. Biotechnol Lett 32:973-977. doi: 10.1007/ s10529-010-0248-2".

Finally, in the Table 2 footnote, "according to (Heijnen 1981)" should be replaced as "according to Heijnen (1981)".

The online version of the original article can be found under doi:10.1007/s10482-017-0940-5.

O. Dias · I. Rocha - E. C. Ferreira

Centre of Biological Engineering, University of Minho,

Campus de Gualtar, 4710-057 Braga, Portugal

O. Dias · T. O. Basso $(\bowtie) \cdot$ A. K. Gombert

Department of Chemical Engineering, Polytechnic

School, University of São Paulo, Av. Prof. Luciano

Gualberto 380, São Paulo, SP 05508-010, Brazil

e-mail: thiagobasso@usp.br

A. K. Gombert

School of Food Engineering, University of Campinas, Rua

Monteiro Lobato 80, Campinas, SP 13083-862, Brazil 